Präv Gesundheitsf 2021 · 16:104-109 https://doi.org/10.1007/s11553-020-00802-z Eingegangen: 29. April 2020

Angenommen: 18. Juni 2020

Online publiziert: 15 . Juli 2020

(c) Der/die Autor(en) 2020

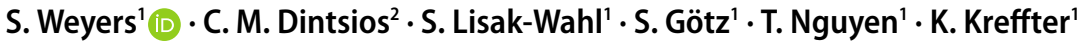

'Institut für Medizinische Soziologie, Universitätsklinikum AÖR - Centre for Health and Society (CHS), Medizinische Fakultät, Heinrich-Heine-Universität Düsseldorf, Düsseldorf, Deutschland ${ }^{2}$ Institut für Versorgungsforschung und Gesundheitsökonomie, Centre for Health and Society, Medizinische Fakultät, Heinrich-Heine-Universität Düsseldorf, Düsseldorf, Deutschland

\section{Ist Bewegung eine Frage des Geldes? Eine Analyse des Kostengeschehens kommunaler Prävention für Kinder aus Nutzerperspektive}

32, 35]. Im Zusammenhang mit diesem sog. Präventionsparadoxon wird davon ausgegangen, dass durch die Vernachlässigung von Alltagspraktiken, Vorlieben und Einstellungen benachteiligter Gruppen bei der Planung von Präventionsmaßnahmen deren Distanz zu Bildungsund Vorsorgeangeboten bekräftigt wird [10].

Aus der Literatur gehen jedoch auch andere Aspekte hervor. In einer systematischen Übersichtsarbeit identifizierten Somerset und Hoare [27] Kosten und Zeit als häufige Barrieren für kindlichen Sport. Eine US-amerikanische Umfrage einkommensschwacher Eltern ermittelte Kosten und Sicherheit als häufigste Barrieren für die Nutzung von Bewegungsangeboten [9]. Somit stellt sich die Frage, ob derartige Aspekte auch im deutschen System von Bewegungsangeboten zum Tragen kommen. Hier spielen freigemeinnützige Träger eine wichtige Rolle, da sie nicht gewinnorientiert arbeiten und Ermäßigungen für Familien in schwierigen Lebenslagen gewähren. Bei Befragungen deutscher Eltern zur Angebotsnutzung im Bereich Früher Hilfen waren die Kosten jedoch ebenfalls zentraler Faktor [26]. Bei einer eigenen Befragung von Eltern mit niedriger Bildung waren Interesselosigkeit, Zeit und Kosten die häufigsten Gründe der Nichtteilnahme an Bewegungsangeboten [36]. Bei allen genannten Studien handelt es sich allerdings um subjektive Angaben der Befragten. Damit bleibt die Frage offen, wie sich das "objektive“ Kostengeschehen aus Nutzerperspektive im $\mathrm{Zu}$ sammenhang mit Bewegungsförderung gestaltet.

Bei der Kostenevaluation aus Nutzerperspektive werden meist Kostenarten wie Medizinprodukte und Dienstleistungen zur Krankheitsprävention oder -behandlung, Zuzahlungen oder Zusatzversicherungen, Fahrtkosten oder Einkommenseinbußen betrachtet [31]. Kostenevaluationen aus Nutzerperspektive zur kommunalen Bewegungsförderung gibt es unseres Wissens im deutschen Kontext bisher nicht. Unter Berücksichtigung der sozialen Ungleichheit stellt sich darüber hinaus die Frage nach der Erschwinglichkeit von Angeboten, d.h. in welcher Relation die vom Nutzer zu tragenden Kosten zum Einkommen stehen. Dabei wird in der Regel das frei verfügbare Einkommen berücksichtigt, da Ausgaben für Wohnen, Bekleidung, Nahrungsmittel und Verkehr essentiell sind und nur das nach Abzug dieser Ausgabenblöcke übrigbleibende Einkommen für persönliche Gesundheitsausgaben zur Verfügung steht [25]. In einer Studie von Bremer [5] war die finanzielle Belastung durch persönliche Gesundheitsausgaben bei Personen mit niedrigem Einkommen vergleichsweise hoch. Die Erschwinglichkeit von An- 


\begin{tabular}{|c|c|c|c|}
\hline Anbieter & $\begin{array}{l}\text { Regulärer Preis bzw. } \\
\text { Preisspanne in } € / h\end{array}$ & $\begin{array}{l}\text { Regulärer Preis bzw. } \\
\text { Preisspanne in } € / \text { Monat }\end{array}$ & Ermäßigung (\%) \\
\hline 1 & $3,33-5,33$ & $20,00-32,00$ & $25 ; 2$. Kind kostenfrei \\
\hline 2 & $3,33-4,00$ & 20,00 & $\begin{array}{l}\text { z. T. Kostenübernahme von } \\
\text { Familienzentren; 30; } 2 \text {. Kind } \\
\text { ermäßigt }\end{array}$ \\
\hline 3 & $4,05-4,76$ & $24,29-28,75$ & $20-30$ \\
\hline 4 & $4,21-5,28$ & $25,26-31,69$ & $33-50$ \\
\hline 5 & $7,33-8,00$ & $44,00-48,00$ & 10 \\
\hline
\end{tabular}

\begin{tabular}{l|l|l|l|}
\hline $\begin{array}{l}\text { Tab. } 2 \\
\text { Anbieter }\end{array}$ & $\begin{array}{l}\text { Regulärer Preis bzw. Preis- } \\
\text { spanne in } \boldsymbol{\epsilon} / \mathbf{h}\end{array}$ & $\begin{array}{l}\text { Regulärer Preis bzw. Preis- } \\
\text { spanne in } \boldsymbol{€} / \text { Monat }\end{array}$ & Ermäßigung \\
\hline 1 & $0,50-5,00$ & $4,00-30,00$ & $25 ; 2$. Kind kostenfrei \\
\hline 2 & 4,00 & 16,00 & $30 ; 2$. Kind ermäßigt \\
\hline 3 & $3,08-3,28$ & $18,00-19,69$ & $20-30$ \\
\hline 4 & $0,00-4,93$ & $16,40-25,20$ & $33-50$ \\
\hline 5 & 14,67 & 44,00 & 10 \\
\hline 6 & $2,76-4,00$ & 16,00 & $\begin{array}{l}\text { Individuelle Abspra- } \\
\text { chen }\end{array}$ \\
\hline
\end{tabular}

geboten zur Bewegungsförderung ist unseres Wissens bisher ebenfalls noch nicht untersucht worden.

Die vorliegende Untersuchung soll daher zwei Fragen beantworten: Welche Kosten müssen Eltern erbringen, um Bewegungsangebote für Kinder wahrzunehmen? Wie erschwinglich sind diese Ausgaben für sozioökonomisch benachteiligte Familien? Diese Frage wird anhand der typischen Angebotsstruktur von Düsseldorf, einer Großstadt mit über 600.000 Einwohnern, beantwortet.

\section{Methodik}

Grundlage für die Untersuchung ist eine Bestandsaufnahme kommunaler Präventionsangebote für Kinder bis sechs Jahre [11], die im Rahmen der Studie "Gesundheit bei Schuleingang" [34] durchgeführt wurde. Aus dieser Bestandsaufnahme wurden mit bewegungsrelevanten Suchbegriffen alle Angebote der sechs größten freigemeinnützigen Träger zur Bewegungsförderung in drei Kategorien herausgefiltert: ElternBaby-Kurse bis ein Jahr, Eltern-KindKurse von ein bis sechs Jahren sowie Wasser- und Schwimmangebote. Zu den resultierenden 36 Angeboten wurden zwischen Juni und September 2019 im
Internet, in Programmheften oder mittels persönlicher Ansprache folgende Daten recherchiert: Gesamtpreis des Angebots, Anzahl der Kurstage, Häufigkeit des Kurses pro Monat, Dauer der Kurseinheit in Minuten, Ermäßigungen und sonstige Charakteristika.

Da die Kurseinheiten unterschiedlich lang sind, wurde für eine bessere Vergleichbarkeit der Preis pro Kurseinheit berechnet (Gesamtpreis/Anzahl Kurstage) und auf dieser Basis der Preis pro Zeitstunde (Kosten pro Kurseinheit/Dauer der Kurseinheit in $\min \times 60)$ und der Preis pro Monat (Kosten pro Kurseinheit $\times$ Häufigkeit des Kurses pro Monat).

Die Erschwinglichkeit für eine sozioökonomisch benachteiligte Familie wurde exemplarisch am Beispiel einer alleinerziehenden Familie mit einem 3-jährigen Kind berechnet, die in einem Düsseldorfer Stadtteil mit besonderem Entwicklungsbedarf lebt und ALG II bezieht. Die Einkommensverhältnisse wurden anhand des Regelbedarfs bestimmt [7] und ergaben einen Gesamtbedarf von $1.377 €$. Die Berechnung der monatlichen Ausgaben erfolgte anhand der durchschnittlichen Mietpreise in dem gegebenen Stadtteil und der für diese Familie durchschnittlichen Konsumausgaben [29], womit sich Fixkosten in Höhe von
$1.177 €$ ergaben. Die Differenz von Gesamtbedarf und Fixkosten resultierte in einem frei zur Verfügung stehenden Einkommen von $200 €$. Für die Bestimmung der Erschwinglichkeit wurden das günstigste und das teuerste Angebot nach Ermäßigung in Relation zum frei verfügbaren Einkommen gesetzt.

\section{Ergebnisse}

Nachfolgend werden die Angebote der sechs verschiedenen Anbieter pseudonymisiert dargestellt. Getrennt für die drei Angebotskategorien werden jeweils die regulären Preise pro Zeitstunde und pro Monat aufgeführt, darüber hinaus Angaben zur Ermäßigung.

Im Bereich ,Eltern-Baby-Kurse' offerieren fünf freigemeinnützige Anbieter insgesamt 13 Angebote wie Babymassagen oder Krabbelgruppen mit meist 90-minütiger Dauer. Anbieter 1 beispielsweise bietet drei verschiedene Kurse an und hat eine Preisspanne von 3,33 bis $5,33 €$ pro Kursstunde. Somit ergeben sich monatliche Kosten von 20,00 bis $32,00 €$. Personen in schwierigen Lebenslagen erhalten eine Ermäßigung von $25 \%$ und das zweite Kind kann kostenfrei teilnehmen. In diesem Preisgefüge bewegen sich auch die anderen Anbieter mit Ausnahme von Anbieter 5, bei dem die Preisspanne pro Stunde und pro Monat deutlich höher liegt und weniger Ermäßigung gewährt wird (• Tab. 1).

Für den Bereich „Eltern-Kind-Kurse" wurden 15 Angebotsvarianten zum Bewegen, Tanzen oder Spielen mit einer Kursdauer zwischen 45 und $180 \mathrm{~min}$ identifiziert. Die Preise liegen zwischen 4,00 und 44,00€/Monat. Ein besonderes Angebot macht Anbieter 1, der an manchen Standorten über einen Gutschein von einer Schwangerschaftsberatungsstelle finanziert wird, sodass lediglich ein symbolischer Betrag von $50 \mathrm{Cent} / \mathrm{h} \mathrm{zu}$ entrichten ist. In einem Stadtteil macht Anbieter 1 außerdem ein Angebot, welches 2- bis 3-mal pro Woche stattfindet und einen Monatspreis von 105,00€ aufweist. Dieser "Ausreißer" wurde in den Analysen nicht berücksichtigt (• Tab. 2).

Für die Kategorie, Wasser-/Schwimmangebote 'wurden von drei Anbietern insgesamt acht verschiedene wöchentli- 
Präv Gesundheitsf 2021 • 16:104-109 https://doi.org/10.1007/s11553-020-00802-z

(c) Der/die Autor(en) 2020

\section{S. Weyers · C. M. Dintsios · S. Lisak-Wahl · S. Götz · T. Nguyen · K. Kreffter}

\section{Ist Bewegung eine Frage des Geldes? Eine Analyse des Kostengeschehens kommunaler Prävention für Kinder aus Nutzerperspektive}

\section{Zusammenfassung}

Hintergrund. Kinder mit niedrigem

Sozialstatus haben häufiger motorische

Defizite, gleichzeitig nehmen sie Angebote

zur Bewegungsförderung seltener wahr. Die

Gründe dafür sind unklar. Im Zusammenhang

mit finanziellen Barrieren ist das objektive

Kostengeschehen aus Nutzerperspektive

bisher kaum erforscht, insbesondere nicht im

Hinblick auf soziale Ungleichheit.

Ziel der Arbeit. Die Studie soll zwei Fragen beantworten: Welche Kosten müssen Eltern

für die Bewegungsangebote ihrer Kinder erbringen? Wie erschwinglich sind diese

Angebote für sozioökonomisch benachteiligte

Familien?

Material und Methoden. Zunächst wurden

36 Angebote freigemeinnütziger Träger in
Düsseldorf für Kinder bis 6 Jahre identifiziert.

Zu diesen Angeboten wurde jeweils der Preis pro Kurseinheit, pro Zeitstunde und pro Monat berechnet. Für die Bestimmung der Erschwinglichkeit wurden das günstigste und das teuerste Angebot nach Ermäßigung in Relation zum frei verfügbaren Einkommen einer alleinerziehenden Familie mit Arbeitslosengeld(ALG)-II-Bezug gesetzt. Ergebnisse. Preise für Eltern-Baby-Kurse rangieren zwischen 20,00 und $48,00 €$ pro Monat, für Eltern-Kind-Kurse zwischen 4,00 und $44,00 €$ und für Wasser- und Schwimmangebote zwischen 18,00 und 28,86€. Ermäßigte Kurspreise können zwischen 4,10 und $21,60 \%$ des frei zur Verfügung stehenden
Einkommens einer alleinerziehenden Familie mit ALG-II-Bezug ausmachen.

Diskussion. Kommunale Bewegungsangebote werden an die finanziellen Bedürfnisse von Familien angepasst. Für sozioökonomisch benachteiligte Familien sind sie jedoch nicht immer erschwinglich. Weitere Ermäßigungen bei ausgewählten Angeboten könnten die Inanspruchnahme von Kindern mit niedrigem Sozialstatus an Bewegungsangeboten erhöhen.

\section{Schlüsselwörter}

Kindergesundheit · Körperliche Aktivität . Gesundheitsförderung · Soziale Ungleichheit

\section{Is physical activity a question of money? An analysis of the costs of community prevention for children from a user's perspective}

\section{Abstract}

Background. Children with low socioeconomic status have an increased risk of motor deficits. At the same time they participate less often in offers to promote physical activity. The reasons for this are unclear. In the context of financial barriers, the objective costs from a user's perspective have not yet been explored, especially not with regard to social inequalities.

Objectives. The study has two aims: What do parents have to pay for the physical activity offers their children participate in? How affordable are these offers for socioeconomically disadvantaged families?
Materials and methods. First, we identified 36 offers of community nonprofit organisations for children up to 6 years in the city of Dusseldorf. For these offers, we calculated the price per unit, per hour and per month. In order to calculate affordability we put the cheapest and the most expensive offer after discount in relation to the disposable income of a single-parent family on social benefits. Results. Prices for parent-baby courses range from 20.00 to $48.00 €$ per month, for parent-child courses they range from 4.00 to $44.00 €$ per month and for swimming courses from 18.00 to $28.86 €$. Prices after discount can account for 4.10 to $21.60 \%$ of the disposable income of a single-parent family with social benefits.

Conclusions. Community nonprofit offers to promote children's physical activity are adapted to families' financial needs. They are, however, not always affordable for socioeconomically disadvantaged families. Further discount in selected offers could increase the participation of children with low socioeconomic status in offers to promote physical activity.

\section{Keywords}

Child health · Physical activity $\cdot$ Health promotion - Social inequalities che Varianten von $45 \mathrm{~min}$ identifiziert. Diese beinhalten Angebote für alle Altersgruppen bis sechs Jahre wie ElternBaby-Schwimmen oder Aufbauschwimmen. Die Preise bewegen sich zwischen 18,00 und 28,86€ pro Monat. Es werden z.T. Ermäßigungen gewährt (• Tab. 3).

In einem zweiten Schritt wurden die Preise der verschiedenen Bewegungsangebote mit dem frei verfügbaren Einkommen einer Familie mit finanziellen Herausforderungen in Beziehung gesetzt. - Tab. 4 zeigt die Monatspreise der nach
Ermäßigung teuersten und günstigsten Angebote in jeder Kategorie sowie den Anteil der ermäßigten Preise am frei verfügbaren Einkommen von $200 €$. Bei Eltern-Baby-Kursen bis ein Jahr macht das ermäßigte Angebot von Anbieter 521,6 \% des frei verfügbaren Einkommens aus, wohingegen das ermäßigte Angebot des Anbieters 4 einen Anteil von 6,32\% ausmacht. Bei Eltern-Kind-Kursen von ein bis sechs Jahren macht der Kurs des Anbieters 1 19,8\% des frei verfügbaren Einkommens aus und auch in diesem Fall ist der Kurs des Anbieters 4 mit 4,10\% am günstigsten. Wasserangebote machen $10 \%$ (Anbieter 2) bis 8,69\% (Anbieter 3) am frei verfügbaren Einkommen aus.

\section{Diskussion}

Ziel der Studie war zu ermitteln, welche Kosten Eltern erbringen müssen, um Bewegungsangebote für Kinder wahrzunehmen und wie erschwinglich diese Ausgaben für Familien mit finanziellen Herausforderungen sind. Dies wurde an- 


\begin{tabular}{|l|l|l|l|}
\hline Tab. 3 & Preise für Wasser-/Schwimmangebote $(n=8$ Angebote) & \\
\hline Anbieter & $\begin{array}{l}\text { Regulärer Preis bzw. } \\
\text { Preisspanne in } \boldsymbol{\epsilon} / \mathbf{h}\end{array}$ & $\begin{array}{l}\text { Regulärer Preis bzw. Preis- } \\
\text { spanne in } \boldsymbol{\epsilon} / \text { Monat }\end{array}$ & Ermäßigung (\%) \\
\hline 2 & $6,00-6,67$ & $18,00-20,00$ & 2 Kind ermäßigt \\
\hline 3 & $8,28-9,62$ & $24,83-28,86$ & $20-30$ \\
\hline 4 & $7,55-7,80$ & $22,66-23,40$ & Bis 20 \\
\hline
\end{tabular}

\section{Tab. 4 Erschwinglichkeit der Angebote}

\begin{tabular}{|c|c|c|c|}
\hline Angebot & $\begin{array}{l}\text { Regulärer } \\
\text { monatli- } \\
\text { cher Preis } \\
(€)\end{array}$ & $\begin{array}{l}\text { Ermäßigter mo- } \\
\text { natlicher Preis } \\
\text { ALG-II-Empfän- } \\
\text { ger } \\
\text { (€) }\end{array}$ & $\begin{array}{l}\text { Anteil ermäßig- } \\
\text { ter Preis am frei } \\
\text { verfügbaren Ein- } \\
\text { kommen (\%) }\end{array}$ \\
\hline \multicolumn{4}{|l|}{ Eltern-Baby-Kurse bis ein Jahr } \\
\hline $\begin{array}{l}\text { TA: Anbieter } 5 \text { "Gut durch das erste } \\
\text { Lebensjahr" } \\
\text { Kaiserswerth, } 90 \mathrm{~min},-10 \%\end{array}$ & 48,00 & 43,20 & 21,60 \\
\hline $\begin{array}{l}\text { GA: Anbieter } 4 \text { „Eltern-Baby-Grup- } \\
\text { pen im ersten Lebensjahr, Spiel für } \\
\text { die Sinne }{ }^{\prime \prime \prime} \\
\text { Stadtmitte, } 90 \text { min, }-50 \%\end{array}$ & 25,26 & 12,63 & 6,32 \\
\hline \multicolumn{4}{|l|}{ Eltern-Kind-Kurse ein bis sechs Jahre } \\
\hline $\begin{array}{l}\text { TA: Anbieter } 5 \text { "Bewegung und } \\
\text { Spaß" } \\
\text { Kalkum, } 45 \mathrm{~min},-10 \%\end{array}$ & 44,00 & 39,60 & 19,80 \\
\hline $\begin{array}{l}\text { GA: Anbieter } 4 \text { „Turnen und Bewe- } \\
\text { gungsspiele für Eltern und Kinder } \\
\text { ab 1,5 Jahren“ } \\
\text { Wersten, } 60 \mathrm{~min},-50 \%\end{array}$ & 16,40 & 8,20 & 4,10 \\
\hline \multicolumn{4}{|l|}{ Wasser-/Schwimmangebote } \\
\hline $\begin{array}{l}\text { TA: Anbieter } 2 \text { „Eltern-Kind-Schwim- } \\
\text { men für Eltern mit Kindern von } \\
\text { 1-3 Jahren" } \\
\text { Mörsenbroich, } 45 \text { min }\end{array}$ & 20,00 & Keine Ermäßigung & 10,00 \\
\hline $\begin{array}{l}\text { GA: Anbieter } 3 \text { „Anfängerschwim- } \\
\text { men für Kinder ab dem } 5 \text {. Lebens- } \\
\text { jahr“ } \\
\text { Bilk, } 45 \text { min, }-20 \%\end{array}$ & 24,83 & 17,38 & 8,69 \\
\hline
\end{tabular}

hand der Angebote freigemeinnütziger Träger berechnet. Die Kosten wurden dem frei verfügbaren Einkommen einer Familie mit finanziellen Herausforderungen gegenübergestellt.

Insgesamt zeigt sich, dass es viele Anbieter und eine Bandbreite an Preisen gibt. Für Eltern-Baby-Kurse bis ein Jahr müssen Eltern zwischen 20,00 und $48,00 € /$ Monat ausgeben. Für ElternKind-Kurse ein bis sechs Jahre müssen Eltern zwischen 4,00 und 44,00€/ Monat entrichten. Bei den Wasser- und Schwimmangeboten gibt es weniger Anbieter und eine geringere Preisvarianz mit 18,00 bis $28,86 € /$ Monat. Ein erster Hinweis in Bezug auf die Erschwing- zur Verfügung stehenden Einkommens von $200 €$ ausmacht.

Die große Varianz der Preise kann dadurch erklärt werden, dass sie an die jeweilige Sozialstruktur im Stadtteil angepasst werden. Dies zeigt sich auch in - Tab. 4, in der die günstigsten Angebote in Stadtteilen mit Entwicklungsbedarf vorgehalten werden. Hier spielen die lokalen Verbände gemeinnütziger Träger der freien Wohlfahrtpflege eine Rolle, die als eingetragene Vereine staatliche Zuwendung und Eigenmittel nutzen, um zum Aufbau sozialer Infrastrukturen beizutragen [4]. Dabei können gerade solche lokalen Akteure die Bedürfnisse in den Lebenswelten prüfen und örtliche Ressourcen zur Gesundheitsförderung bündeln [19]. Dies wird bei vielen der hier analysierten Bewegungsangeboten dadurch sichtbar, dass Anbieter mit Familienzentren kooperieren und die Preisgestaltung deutlich anpassen.

Nach den Leistungen für Bildung- und Teilhabe [20] können Eltern mit ALGII-Bezug für Kinder unter 18 Jahren außerdem monatlich $15 €$ für die Teilhabe am sozialen und kulturellen Leben wie z. B. Mitgliedschaft in einem Sportverein beantragen. Damit wären die Kosten einiger ermäßigter Eltern-Baby- oder Eltern-Kind-Kurse abgedeckt. Von Vorteil ist auch, dass für keine der ermittelten Kursvarianten aufwändige Materialkosten wie spezielle Sportausrüstungen oder -bekleidung erforderlich sind und lediglich Utensilien wie Handtücher oder Sportschuhe benötigt werden.

Allerdings könnten Fahrtkosten entstehen, die den weiteren Aufwendungen für Bewegungskurse zugerechnet werden müssten. Kinder bis 6 Jahre fahren zwar umsonst im öffentlichen Personennahverkehr. Für Düsseldorfer Eltern, die Sozialleistungen beziehen, kostet ein Ticket jedoch 39,35€/Monat [21]. Darüber hinaus konkurrieren die hier ermittelten Kosten für Bewegungskurse mit anderen Kosten für Freizeit, Unterhaltung und Kultur.

Eine englische Studie zur Zahlungsbereitschaft bei Sport und Bewegung ergab, dass Befragte durchschnittlich $27 £ /$ Monat (in 2012 ca. $33 €$ ) für Sport und Bewegung ausgeben [1]. In einer deutschen Studie beläuft sich die Zahlungsbe- 
reitschaft von Eltern für Präventionsangebote von kindlichem Übergewicht auf $23,04 €$ pro Monat bei denjenigen, die überhaupt zahlungsbereit sind, und auf $10,27 €$ in der Gesamtstichprobe [17]. Ein besonderer Schwerpunkt auf sozioökonomisch benachteiligte Familien wurde in diesen Studien jedoch nicht gelegt. Bei den möglichen Reduktionen liegen die hier ermittelten Monatspreise zwischen $8,20 €$ und $17,38 €$ über der von [17] ermittelnden konservativen Zahlungsbereitschaft von $10,27 €$.

Könnte eine Preisreduktion die Zahlungsbereitschaft und somit die Teilnahme an Bewegungsangeboten steigern? Subjektiv stimmen Eltern und Erzieher dieser Vermutung zu $[9,18,30]$. Auch objektiv scheinen sich finanzielle Anreize positiv auf die Teilnahme an Präventions- oder "Lifestyle-Programmen“ auszuwirken. So steigerte die Anreizbedingung "Bezahlung“ die Teilnahme einkommensschwacher Eltern an einem Kurs zur Förderung verhaltensauffälliger Kinder [14]. Ebenso wurden Bonusprogramme zur Bewegungsförderung der Gesetzlichen Krankenkassen deutlich häufiger von den Versicherten genutzt als selbst zu zahlende Angebote [23]. Eine englische Studie fand eine erhöhte sportliche Aktivität bei der Senkung von Teilnahmekosten, v. a. bei Schwimmangeboten [1]. Den Autoren zufolge sollten Aktivitäten gezielt bezuschusst werden. Dies erscheint deswegen sinnvoll, weil in der vorliegenden Arbeit Schwimmkurse vergleichsweise teuer waren und in einer eigenen Elternbefragung sich hier auch die größten sozialen Ungleichheiten bei der Nutzung ergaben [35].

\section{Limitationen}

Unseres Wissens ist dies die erste Studie, welche eine objektive Bewertung der Kosten und Erschwinglichkeit kommunaler Bewegungsförderung für Kinder vornimmt und dabei einen Schwerpunkt auf sozioökonomisch benachteiligte Familien legt. Dabei haben wir exemplarisch nur die Angebote der wichtigsten freigemeinnützigen Träger dargestellt, dies jedoch in einer Vollerhebung. Angebote der privatwirtschaftlichen Träger beispielsweise wurden nicht berücksichtigt.

Bei der Berechnung der Erschwinglichkeit orientierten wir uns an den Vorgaben des National-Quality-Forums [25] und zogen das frei verfügbare Einkommen heran. In einer Studie zur Erschwinglichkeit gesunder Ernährung für Kinder mit Hartz-IV-Bezug wurden die Ausgaben für optimierte Mischkost hingegen mit dem Regelsatz für Nahrungsmittel in Bezug gesetzt. Mit dem Ergebnis, dass der Tagessatz in HartzIV-Haushalten nicht ausreicht, um Kinder gesund zu ernähren [16]. Berechnet man die Erschwinglichkeit in der hier vorliegenden Studie analog und setzt man die Preise in Bezug zum Regelsatz für Freizeit, Unterhaltung und Kultur für Kinder bis 5 Jahre, wären im günstigsten Fall 23,21\% des Budgets verplant. Auch unter Zuhilfenahme von Leistungen für Bildung und Teilhabe (BuT) wäre es nicht möglich, die teureren Kurse aus diesem Budget heraus zu bezahlen. In der vorliegenden Studie wurde jedoch von dieser Vorgehensweise abgesehen, da Individuen zur Deckung ihrer Ausgaben die in der Bedarfsrechnung zugewiesenen Beträge aus verschiedenen Gruppen kombinieren können. Die Erschwinglichkeitsbewertung über das frei verfügbare Einkommen scheint daher geeigneter, individuellen Ausgabepräferenzen Raum zu lassen.

\section{Fazit für die Praxis}

- Dem Faktor Kostengestaltung kommt
eine entscheidende Rolle bei der
Bereitschaft zur Teilnahme an Bewe-
gungsangeboten für Kinder zu.
— In den Kommunen wird bereits
viel getan, um die Angebote an
die finanziellen Bedürfnisse von
Familien anzupassen. Dennoch
ergeben sich Hinweise, dass die
Zahlungsbereitschaft der Eltern
immer noch überschritten wird
und Angebote für sozioökonomisch
benachteiligte Familien nicht ohne
Weiteres erschwinglich sind.
- Stärkere Ermäßigungen bei ausge-
wählten Angeboten sind daher ein
sinnvoller Weg die Inanspruchnahme
solcher Familien zu erhöhen.

\section{Korrespondenzadresse}

Dr. phil. S. Weyers, MME

Institut für Medizinische Soziologie, Universitätsklinikum AÖR - Centre for Health and Society (CHS), Medizinische Fakultät, Heinrich-Heine-Universität Düsseldorf Moorenstraße 5, 40225 Düsseldorf, Deutschland

weyerss@uni-duesseldorf.de

Danksagung. Wir danken Dr. Helen Salia für die Überlassung der Daten.

Funding. Open Access funding provided by Projekt DEAL.

\section{Einhaltung ethischer Richtlinien}

Interessenkonflikt. S. Weyers, C.M. Dintsios, S. LisakWahl, S. Götz, T. Nguyen und K. Kreffter geben an, dass kein Interessenkonflikt besteht.

Für diesen Beitrag wurden von den Autoren keine Studien an Menschen oder Tieren durchgeführt. Für die aufgeführten Studien gelten die jeweils dort angegebenen ethischen Richtlinien.

Open Access. Dieser Artikel wird unter der Creative Commons Namensnennung 4.0 International Lizenz veröffentlicht, welche die Nutzung, Vervielfältigung, Bearbeitung, Verbreitung und Wiedergabe in jeglichem Medium und Format erlaubt, sofern Sie den/die ursprünglichen Autor(en) und die Quelle ordnungsgemäß nennen, einen Link zur Creative Commons Lizenz beifügen und angeben, ob Änderungen vorgenommen wurden.

Die in diesem Artikel enthaltenen Bilder und sonstiges Drittmaterial unterliegen ebenfalls der genannten Creative Commons Lizenz, sofern sich aus der Abbildungslegende nichts anderes ergibt. Sofern das betreffende Material nicht unter der genannten Creative Commons Lizenz steht und die betreffende Handlung nicht nach gesetzlichen Vorschriften erlaubt ist, ist für die oben aufgeführten Weiterverwendungen des $\mathrm{Ma}$ terials die Einwilligung des jeweiligen Rechteinhabers einzuholen.

Weitere Details zur Lizenz entnehmen Sie bitte der Lizenzinformation auf http://creativecommons.org/ licenses/by/4.0/deed.de.

\section{Literatur}

1. Anokye NK, Pokhrel S, Fox-Rushby J (2014) Economic analysis of participation in physical activity in England: implications for health policy. Int J Behav Nutr Phys Act 11:117

2. Augste C, Jaitner D, Storr U (2012) Schuleingangsuntersuchung offenbart soziale Unterschiede bei Körperkomposition, Bewegungsverhalten und motorischem Entwicklungsstand. Dtsch Z Sportmed 2012(10):305-310

3. Barnett LM, Lai SK, Veldman SLC et al (2016) Correlates of gross motor competence in children and adolescents. A systematic review and metaanalysis. Sports Med 46(11):1663-1688 
4. Bauer R, Dahme H-J, Struck N (2011) Freie Träger. In: Thole W (Hrsg) Grundriss Soziale Arbeit. Ein einführendes Handbuch, 4. Aufl. Bd. 16. Springer VS, Wiesbaden, S 813-829

5. BremerP (2014) Forgone care and financial burden due to out-of-pocket payments within the German health care system. Health Econ Rev 4:36

6. Brophy S, Cooksey R, Lyons RA et al (2011) Parental factors associated with walking to school and participation in organised activities at age 5 . Analysis of the millennium cohort study. BMC Public Health 11:14

7. Bundesministerium für Arbeit und Soziales (2019) Grundsicherung für Arbeitsuchende. Sozialgesetzbuch SGB II. Fragen und Antworten. Bundesministerium für Arbeit und Soziales, Bonn

8. Dollman J, Lewis NR (2010) The impact of socioeconomic position on sport participation among South Australian youth. J Sci Med Sport 13(3):318-322

9. Finkelstein DM, Petersen DM, Schottenfeld LS (2017) Promoting children's physical activity in low-income communities in colorado: what are the barriers and opportunities? Prev Chronic Dis 14:E134

10. Franzkowiak P (2015) Prävention und Krankheitsprävention. BZgA, Köln

11. Götz S, Weyers S, Wahl S (2016) Bestandsaufnahme der kommunalen Präventionsangebote für werdende Eltern und Kinder zwischen 0 und 6 Jahren in Düsseldorf(Unveröffentlichter Bericht)

12. GrafC, Koch B, Dordel S et al (2003) Prävention von Übergewicht und Adipositas durch körperliche Aktivität - eine familiäre Aufgabe? Aktuel Ernahrungsmed. https://doi.org/10.1055/s-2003816326

13. Hardy LL, O'Hara BJ, Rogers K et al (2014) Contribution of organized and nonorganized activity to children's motor skills and fitness. J Sch Health 84(11):690-696

14. Heinrichs N (2006) The effects of two different incentives on recruitment rates of families into a prevention program. J Primary Prevent 27(4):345-365

15. Hilpert M, Brockmeier K, Dordel S et al (2017) Sociocultural influence on obesity and lifestyle in children. A study of daily activities, leisure time behavior, motor skills, and weight status. Obes Facts 10(3):168-178

16. Kersting M, Clausen K (2007) Wie teuer ist eine gesunde Ernährung für Kinder und Jugendliche? Ernährungsumschau 9:508-513

17. Kesztyüs D, Lauer R, Schreiber AC et al (2014) Parents' willingness to pay for the prevention of childhood overweight and obesity. Health Econ Rev 4(1):20

18. Kreffter K, Wahl S, Dragano N et al (2019) Familien mit Bedarf sind Familien, auf die wir zugehen müssen. Prävention Z Gesundheitsförd 25(3):263

19. Landesgesundheitsamt Baden-Württemberg (2015) Handbuch zur Kommunalen Gesundheitsförderung. Landesgesundheitsamt BadenWürttemberg, Stuttgart

20. Landeshauptstadt Düsseldorf (2020) Bildung und Teilhabe. https://www.duesseldorf.de/soziales/ bildungs-und-teilhabepaket.html. Zugegriffen: 13. Jan. 2020

21. Landeshauptstadt Düsseldorf (2020) SozialTicket. https://www.duesseldorf.de/soziales/sozialticket. html. Zugegriffen: 13. Jan. 2020

22. Landeszentrum Gesundheit Nordrhein-Westfalen (2019) Monitoring Kindergesundheit. Reports der Schuleingangsuntersuchungen. Report 2016
23. LangeC,ZieseT(2011)Daten und Fakten:Ergebnis se der Studie "Gesundheit in Deutschland aktuell 2009". Beiträge zur Gesundheitsberichterstattung des Bundes. Robert-Koch-Institut, Berlin

24. Manz K, Schlack R, Poethko-Müller C et al (2014) Körperlich-sportliche Aktivität und Nutzung elek tronischer Medien im Kindes- und Jugendalter. Ergebnisse der KiGGS-Studie - Erste Folgebefragung (KiGGS Welle 1) (Physical activity and electronic media use in children and adolescents: results of the KiGGS study: first follow-up (KiGGS wave 1)). Bundesgesundheitsblatt Gesundheitsforschung Gesundheitsschutz 57(7):840-848

25. National Quality Forum (2014) Measuring affordability from the patient's perspective. https:// www.qualityforum.org/Publications/2014/09/ Measuring_Affordability_from_the_Patient_s_ Perspective.aspx.Zugegriffen: 13. Jan. 2020

26. Schmolke R, Schmutz E (2015) Bericht zur Planung, Durchführung und Auswertung von Elternforen zur Beteiligung von Familien an der Evaluation und Weiterentwicklung der Frühen Förderung von Familien in Stuttgart

27. Somerset S, Hoare DJ (2018) Barriers to voluntary participation in sport for children: a systematic review. BMC Pediatr 18(1):47

28. Starker A, Lampert T, Worth A et al (2007) Motorische Leistungsfähigkeit. Ergebnisse des Kinder- und Jugendgesundheitssurveys (KiGGS) (Motor Fitness. Results of the German Health Interviewand Examination Survey for Children and Adolescents (KiGGS)). Bundesgesundheitsblatt Gesundheitsforschung Gesundheitsschutz 50(56):775-783

29. Statistisches Bundesamt (2020) Konsumausgaben und Lebenshaltungskosten. Struktur der Konsumausgaben privater Haushalte nach dem monatlichen Haushaltsnettoeinkommen 2017. https://www.destatis.de/DE/Themen/ Gesellschaft-Umwelt/Einkommen-KonsumLebensbedingungen/KonsumausgabenLebenshaltungskosten/Tabellen/listemonatlichen-haushalts-nettoeinkommen.html; jsessionid =5F783A77A39A108DB7921 C9CB8099 B4A.internet712.Zugegriffen: 13. Jan. 2020

30. Stöbe-Blossey S, Hagemann L, Klaudy EK et al (2019) Abschlussbericht „Evaluation Familienzentren NRW"

31. B-WB T, Bae YH, Le QA (2016) A systematic review of health economic evaluation studies using the patient's perspective. Value Health 19(6):903-908

32. Telama R, Laakso L, Nupponen H et al (2009) Secular trends in youth physical activity and parents' socioeconomic status from 1977 to 2005. Pediatr Exerc Sci 21(4):462-474

33. Venetsanou F, Kambas A, Giannakidou D (2015) Organized physical activity and health in preschool age. A review. Cent Eur J Public Health 23(3):200-207

34. Wahl S, Kreffter K, Frölich S et al (2018) Die Schuleingangsuntersuchung als Türöffner für die gesundheitswissenschaftliche Forschung? Eine Analyse zur Studienteilnahme „schwer erreichbarer" Bevölkerungsgruppen. Bundesgesundheitsblatt Gesundheitsforschung Gesundheitsschutz 61(10):1236-1241

35. Weyers S, Kreffter K, Wahl S (2018) Soziale Ungleichheit der Inanspruchnahme kommunaler Bewegungsangebote. Public Health Forum 26(4):365-367

36. Weyers S, Wahl S, Kreffter K (2019) Gesundheit bei Schuleingang - die Bedeutung kommunaler Präventionsketten für vulnerable Kinder (CoLiPre) (Abschlussbericht) 